\title{
¿QUÉ HAY DE MALO EN LA DISCRIMINACIÓN?
}

\author{
Ángel Puyol González \\ Universitat Autònoma de Barcelona
}

RESUMEN. Generalmente, creemos que una discriminación es moralmente mala si está basada en prejuicios, en juicios incorrectos sobre el menor valor moral de las personas. Pero esa creencia tiene dos problemas. Primero, una sociedad liberal no siempre puede reconocer una discriminación basada en prejuicios como moralmente mala. Y segundo, impide ver que algunas discriminaciones moralmente malas no están basadas en prejuicios. Para evitar ambos problemas, hay que definir las discriminaciones moralmente malas por los efectos (efectos sobre la igualdad de oportunidades) que la discriminación causa en las víctimas y no por la razones (prejuicios) de quienes discriminan.

Palabras clave: discriminación, igualdad, justicia, liberalismo.

ABSTRACT. Usually, we think that a discrimination is morally wrong if it is based upon prejudices or biases, the incorrect judgements of lesser moral worth of some persons. But that thought has two problems. First, a liberal society can not always recognize a discrimination based on prejudices as a morally wrong discrimination. And second, the discrimination based on biases hides the fact that some discriminations are not based on biases. So, to avoid both problems, we should define the morally wrong discrimination by their effects (effects on the equality of opportunity) on the victims rather than by the reasons (biases or prejudices) of the discriminators.

Keywords: discrimination, equality, justice, liberalism. 
odos estamos convencidos de que la discriminación es moralmente mala. Pero ¿por qué? La respuesta más habitual es afirmar que la discriminación se fundamenta en prejuicios sobre la inferioridad moral de las personas o los colectivos. Así, la discriminación se asocia a «las desigualdades de trato que son injustas o arbitrarias por basarse en concretas razones especialmente odiosas o rechazables al suponer la negación de la propia igualdad entre los hombres» ${ }^{1}$, o «a los prejuicios, los juicios incorrectos sobre el menor valor moral» ${ }^{2}$. En este artículo se defienden dos tesis. En primer lugar, se sostiene que si la discriminación depende únicamente de los prejuicios morales basados en la inferioridad de individuos o colectivos específicos, las sociedades liberales tienen especiales dificultades para reconocer y, consiguientemente, denunciar y prohibir determinadas formas de discriminación, lo que se puede expresar como la paradoja liberal de la discriminación. En segundo lugar, existen formas de discriminación que no siempre podemos vincular a prejuicios sobre la inferioridad moral, lo que lleva a pensar que no son los prejuicios sino otro elemento de la discriminación lo que la vuelve, en todos los casos, moralmente reprobable. Ese elemento constante tiene que ver con los efectos antes que con las razones de la discriminación.

\section{DISCRIMINACIÓN Y PREJUICIOS}

La lucha contra la discriminación es parte constitutiva de la igualdad de trato que nos debemos unos a otros. Lo es tanto que la misma palabra designa la desigualdad injusta que presuponemos tras ella, sin reparar en que, en origen, se trata de un término meramente descriptivo. Discriminar es distinguir sin más, diferenciar una cosa de otra. Pero la costumbre de vincular el vocablo a la opresión y la humillación que sufren determinados colectivos lo ha convertido en la expresión misma de la denuncia y la inmoralidad. En nuestro vocabulario moral y político, la discriminación consiste en proferir un trato excluyente a individuos o colectivos por motivos racistas, sexistas, clasistas, culturales... Sin embargo, es obvio que si bien hay discriminaciones injustas o inmorales, otras claramente no lo son. Si uno siente un atractivo sexual preferente por personas de su misma etnia, escoge a los amigos por afinidad cultural o de costumbres, selecciona a una ginecóloga porque prefiere confiar la intimidad a una persona de su mismo sexo, favorece a una hija en el negocio familiar, o rechaza a negros y asiáticos para interpretar el papel de Napoleón en una película, no pensamos que se trate necesariamente de una discriminación inmoral. Lo mismo puede ocurrir en el caso de la discriminación inversa o positiva que, pese a que también está relacionada con el sexo, la etnia o la discapacidad, recibe o pretende recibir una justificación moral. Pero otras formas de discriminación, como vetar la entrada de magrebíes y gitanos a las discotecas, poner trabas a las mujeres en la promoción laboral o simplemente sen-

\footnotetext{
${ }^{1}$ Rodríguez Piñero, M., y Fernández LóPez, M. F., 1986: Igualdad y discriminación, Madrid: Tecnos, p. 156; BENGOECHEA GIL, M. Á., 2002: Igualdad, diferencia y probibición de discriminación, Tesis doctoral presentada en la Universidad Carlos III de Madrid, p. 380.

2 AleXAnder, L., 1992: «What makes wrongful discrimination wrong? Biases, preferences, stereotypes, and proxies...», University of Pennsylvania Law Review, vol. 141: 149, p. 219.
} 
tirse molesto porque un inmigrante se ha sentado en el asiento contiguo en el autobús, nos parecen moralmente intolerables. ¿Qué diferencia, pues, a ambos tipos de discriminación? ¿Qué distingue una discriminación injusta de la que no lo es? Sin duda, se trata de una de esas cuestiones que la filosofía se debe plantear, ya que, por habitual y descontada, parece mucho más obvia de lo que en realidad es.

Algunas de las respuestas más comunes a esas preguntas resultan claramente insuficientes. Por ejemplo, hay quien cree que la discriminación es moralmente mala si utiliza rasgos inmodificables para diferenciar el trato a los demás, como el sexo, el color de la piel o una discapacidad. Sin embargo, no nos parece malo impedir que los ciegos piloten aviones, o que sólo se contrate a gitanos como extras en una película dedicada a Carmen Amaya. También hay quien piensa que lo que convierte a la discriminación en mala es que se base en una característica irrelevante de las personas para, por ejemplo, contratarlas laboralmente. El sexo sería claramente irrelevante para ejercer la medicina o la abogacía, lo que volvería moralmente injustificable discriminar por razón de sexo a las personas que desean ingresar o triunfar en esas profesiones. El problema de esa respuesta es que a muchos discriminadores no les parece irrelevante el sexo, el color de la piel u otras características que sus opositores denuncian como tales, porque lo que está en juego es, precisamente, saber qué convierte en irrelevante a esos rasgos en la actividad a desarrollar. Por ejemplo, un empresario puede considerar que la belleza rosada de la juventud, la simpatía ingenua y la delicada habilidad para traer y llevar el café sumisa y cariñosamente son rasgos totalmente relevantes para contratar a una secretaria; o puede estar convencido de que la buena presencia para ser director de banco coincide con personas de cabellera gris y de origen caucásico (lo que excluye al resto de etnias y, por supuesto, a todos los calvos). Por otra parte, los colegios privados con ideario judío pueden pensar que pertenecer al judaísmo es una característica relevante del trabajo de un profesor en sus centros; los dirigentes de la Iglesia católica creen que ser mujer es un atributo definitivamente relevante para prohibir el ejercicio del sacerdocio y el acceso consiguiente a todos los estamentos de poder dentro de la institución religiosa reservados a los sacerdotes; y las asociaciones en defensa del aborto pueden pensar que congregar con la ideología proabortista es relevante para acceder al trabajo administrativo de la organización. Y todo ello a pesar de que resulta muy discutible que la juventud femenina, la cabellera plateada de algunos caucásicos, la adscripción religiosa, el sexo o la ideología que uno profesa, formen parte de la estricta competencia profesional requerida para todas esas ocupaciones.

Ligado a este último argumento, muchos piensan que lo que vuelve inmoral a la discriminación es que, a la hora de juzgar a las personas laboral o académicamente, se tengan en cuenta cuestiones ajenas al mérito. Pero apelar al mérito como la clave de la lucha contra la discriminación comporta algunos contrasentidos. En primer lugar, en el caso del acceso a un cargo público, es plausible mantener la imparcialidad asociada al mérito individual, pero tratándose de empleos privados, en los que los empleadores tienen un amplio margen de definición de lo que debe contar como mérito, las cosas ya no son tan fáciles. Además, a pesar de que es aconsejable que las empresas respeten unos mínimos requisitos de ausencia de discriminación, no resulta obvio que siempre haya que imponerlos. Una gran parte de las promociones laborales dentro de las empresas privadas se establece por confianza, lo que introduce un elemento sub- 
jetivo de evaluación social compleja. No estoy diciendo que no se pueda o no se deba combatir la discriminación en el ámbito laboral privado, sino que el argumento a favor de ese combate no debería depender del principio del mérito, ya que no se trata de un criterio suficientemente objetivo como para saber en todo momento si se respeta o no como debería. El contenido del mérito en el ámbito laboral privado está abierto a ciertas ambigüedades inevitables.

En segundo lugar, el fanatismo y la intolerancia pueden estar detrás de la concepción del mérito. Imaginemos que la mayoría de los hombres no desean que sus jefes sean mujeres, y supongamos que las empresas que no contratan a mujeres en puestos de dirección generan un ambiente laboral más tranquilo y, por tanto, más productivo, lo que las vuelve más competitivas en el mercado. O imaginemos que la clientela de una empresa de servicios prefiere tratar con profesionales que no pertenezcan a etnias diferentes a la mayoritaria. En ambos casos, las preferencias intolerantes influyen en el significado de que cuenta como calificación laboral. Por tanto, nos enredamos en un círculo vicioso si esperamos que el respeto al principio del mérito corrija la discriminación.

En tercer lugar, el mérito no sólo es un criterio poco objetivo, sino que además pone el énfasis de la discriminación en un aspecto ajeno a las personas, puesto que la meritocracia tiene que ver más con la eficiencia que con la igualdad. Si basamos lo injusto de la discriminación en que no hemos juzgado la valía de las personas por sus méritos, lo que estamos haciendo, implícitamente, es desconectar la inmoralidad que hay en la discriminación de la persona discriminada y entregarla a los requisitos impersonales de la eficiencia. Es como si dijéramos: «usted ha sido discriminada porque no se ha respetado su mayor eficiencia». Como es fácil de advertir, éste es el tipo de argumento que seduciría a alguien preocupado exclusivamente por la productividad, pero no necesariamente a alguien comprometido con lo que hace que la discriminación sea mala por sí misma. Fácilmente se podría ampliar la argumentación afirmando que si una acción discriminadora resultase, por la razón que fuese, más eficiente, entonces habría que aprobarla. Y no es difícil imaginar casos en los que eso sucedería. Por ejemplo, si la sucursal de un banco empezase a perder clientela después de haber contratado a un individuo de origen magrebí como cajero, la eficiencia aconsejaría la discriminación. Por tanto, lo que convierte a la discriminación en mala no debería estar ligado al principio del mérito, sino a algún otro factor que sea más objetivo y esté más centrado en la persona discriminada.

Por otra parte, y desde una perspectiva más igualitarista, se podría pensar que lo que resulta incorrecto de la discriminación es que perpetúa el bajo estatus social históricamente condicionado de algunos grupos sociales, como las mujeres, los negros o los gitanos. El problema de ese argumento, no obstante, es que no incluye las discriminaciones hacia personas que no pertenecen a esos grupos históricamente consolidados, como, por ejemplo, los enfermos mentales, los portadores del SIDA, los analfabetos, los parados de larga duración, los ex presidiarios o los nuevos inmigrantes económicos que provienen del este de Europa, de Asia o del norte de África. Y tampoco incluye nuevas formas posibles de discriminación.

Pues bien, ¿qué es lo que vuelve moralmente injustificable la discriminación si no es nada de lo que se ha dicho hasta ahora? La discriminación es una preferencia a 
favor de algunas personas y en contra de otras. Cuando preferimos a unos y no a otros utilizamos diferentes tipos de razones. La simpatía, la lealtad, el atractivo, la familiaridad, la compasión, el sentido del humor, la conexión con los propios gustos e intereses musicales, religiosos, ideológicos, políticos, pueden ser motivos suficientes para preferir a unas personas y no a otras, y no nos parece que ello implique automáticamente una discriminación injusta. También es posible que un individuo busque como pareja a personas de su misma religión, ideología o etnia sin que ello le impida sentirse feliz trabajando, estudiando o yendo en el metro con el resto de personas. En este caso, mantenemos una cierta sospecha sobre su sentido de la discriminación, pero tampoco nos parece claramente condenable. En cambio, otros individuos pueden mostrar un rechazo permanente hacia las personas por su sexo, orientación sexual, etnia, condición social, religión, enfermedad, aspecto o ideología. En estos casos, podemos decir que la discriminación refleja un prejuicio absoluto o universal, mientras que en el anterior diríamos que el tipo de prejuicio que domina es relativo o contextual. Quien cree, sin fundamento racional, consciente o inconscientemente, que las mujeres no son aptas para dirigir una gran organización empresarial, militar o política, mantiene un prejuicio absoluto contra la capacidad de liderazgo de las mujeres en esos ámbitos. Por tanto, tratándose de prejuicios morales absolutos, la discriminación es injustificable. Pero ¿por qué? ¿Todos los prejuicios morales son necesariamente inaceptables?

Algunos tipos de prejuicios no nos parecen tan claramente malos. Hay quien piensa que las personas que poseen mayores virtudes morales, bien por ellas mismas, bien por la posición social que ocupan, merecen un trato especial, en el sentido diferenciador de que merecen mayor respeto y consideración que otras. Por ejemplo, muchos monárquicos piensan que la familia real no debe ser tratada moralmente como a las demás. Muchos católicos piensan de similar modo respecto al Papa. Para ambos colectivos, existe una desigualdad moral entre esos personajes y el resto de la humanidad que se fundamenta en una determinada teoría de la virtud moral que establece desigualdades necesarias.

Existe otro tipo de prejuicios cuya incorrección todavía pasa más inadvertida. Muchas personas tienen la convicción de que es moralmente recomendable, o incluso obligatorio, mostrar más preocupación por el bienestar de los amigos, la familia, la tribu o la comunidad nacional que por el resto de mortales. En esos casos, el prejuicio que sustenta a las actitudes discriminatorias no sólo no produce indignación, sino que a menudo provoca el elogio. La mayoría de nosotros consideraríamos un defecto moral no demostrar mayor desvelo por los hijos propios que por los del vecino, y también entendemos que se exprese mayor interés por la suerte de los miembros de la propia comunidad que por el resto. Cuando sucede una catástrofe en un lugar turístico alejado de nuestras fronteras, todos los informativos nacionales comienzan la noticia mostrando una especial inquietud por el paradero de los suyos, y ante una competición deportiva internacional, casi nadie censura el deseo, incluso públicamente manifestado, de que ganen los del propio país. Otra clase de prejuicios vinculada al bienestar prioritario de los más allegados resulta moralmente más difusa, como un determinado tipo de patriotismo que defiende un estado del bienestar reservado para los nacionales (consideraciones de eficiencia al margen), o el prejuicio contextual cada día más extendido de quienes se quejan amargamente de que los inmigrantes extran- 
jeros acaparen sistemáticamente las ayudas escolares, sociales y de acceso a la vivienda reservadas a los más pobres.

Así pues, no todos los prejuicios son igualmente detestables desde un punto de vista moral, aunque es controvertible saber dónde hay que poner los límites. Sin embargo, se puede argumentar que es perfectamente compatible preferir a las personas moralmente virtuosas o a los allegados sin sentir menosprecio por los demás, pese a que exista una tendencia casi espontánea en muchas personas a conectar el interés especial por lo propio con la inferioridad de lo ajeno. Los amigos, la familia y los vecinos no son moralmente más valiosos que los demás, pero merecen una preocupación especial por nuestra parte. Por otro lado, se puede esgrimir que no es igualmente malo actuar preferentemente hacia un grupo de individuos desatendiendo al mismo tiempo al resto de la humanidad que perjudicar directamente a un grupo social. El primer caso representa una extensión lógica de los compromisos morales hacia ciertas personas y colectivos, mientras que el segundo suele presuponer una ideología que proclama que los miembros del grupo directamente perjudicado son moralmente inferiores o menos valiosos, bien en conjunto - como los judíos para los nazis-, bien a partir de algún atributo o capacidad particular. Hay que señalar, sin embargo, que, desde el punto de vista de la persona discriminada, esa última distinción es absurda, ya que sus atributos personales, reconocidos o negados, suelen formar parte de su identidad. Éste el caso de la discriminación contra la mujer cuando el discriminador argumenta, falazmente, que, en realidad, no discrimina a las mujeres, sino sólo a su capacidad de liderar organizaciones complejas. Negar a las mujeres o a cualquiera, sin una teoría contrastada, su capacidad igual a los demás es como negar su sexo, su presencia física o su origen familiar: es negarlos sin más, denigrarlos como personas, admitir su inferior valor moral como seres humanos.

Se podría pensar que hemos entrado de lleno en la respuesta adecuada a la cuestión de lo que hace que la discriminación sea moralmente mala. Toda discriminación sospechosa presupone un prejuicio, pero es especialmente dañina cuando se trata de un prejuicio absoluto o universal ${ }^{3}$ y cuando éste supone una creencia errónea en la inferioridad de determinadas personas o colectivos para ser lo que otros pueden ser o desempeñar actividades que otros pueden desempeñar.

Cabe resaltar que los efectos de la discriminación van más allá del daño directo que se inflige a las personas discriminadas. Hay un daño indirecto que tiene que ver con la imagen que los discriminados tienen o elaboran de sí mismos tras la costumbre de vivir bajo el yugo de la discriminación. Por efecto de las llamadas preferencias adaptativas, que todas las personas construimos como reacción natural - y racionalal contexto de limitación de recursos en que estamos acostumbradas a vivir, los discriminados pueden acabar adoptando la imagen de sí mismos que sus discriminadores proyectan sobre ellos, lo que explica que muchas mujeres lleguen al falso convencimiento de que son menos capaces que los hombres para realizar las tareas atribuidas tradicionalmente a ellos, o que miembros de minorías históricamente discriminadas acaben creyendo que nunca estarán lo bastante preparadas para alcanzar determinadas metas sociales. La paradoja del esclavo feliz ilustra la trampa que las preferencias

3 Cfr. AleXander, 1992. 
adaptativas construyen, inconscientemente, en las personas que reciben una discriminación permanente ${ }^{4}$.

Algunas formas de discriminación son fruto de actitudes conscientes que se basan en teorías bien sobre la superioridad biológica de unos grupos sobre otros (los hombres sobre las mujeres, los blancos sobre los negros, los arios sobre los judíos y los gitanos...) bien sobre la superioridad cultural (los europeos sobre los africanos, los occidentales sobre el resto de la humanidad...). No hace falta remontarse muy atrás para encontrar ambos tipos de teorías. El famoso y polémico libro de Richard HERNSTEIN y Charles MurRAY, The Bell Curve, escrito en 1994, sugiere que los negros son genéticamente menos inteligentes que los blancos. Michael Levin, en Why Race Matters (1997), añade que los negros no sólo son menos inteligentes, sino que además tienen una tendencia innata a la agresividad y al crimen superior a la de los blancos. Steven GoldBerg, en Why Men Rule: A Theory of Male Dominante (1993), sostiene que las diferencias biológicas entre los sexos explican la inevitable dominación social de los hombres, ya que poseen un nivel de testosterona superior a las mujeres que les proporciona mayor agresividad, competitividad y ambición que ellas. Respecto a la superioridad cultural de unos grupos sobre otros, existen teorías que atribuyen el bajo estatus social de los negros de Estados Unidos a una especie de patología cultural, que proviene de una ética del trabajo muy poco competitiva en el mercado, un desinterés crónico por el esfuerzo académico y una dificultad superior a otras culturas étnicas por buscar y encontrar trabajo. Algo parecido a la imagen que los gitanos tienen en España todavía para muchas personas. Los estadounidenses de origen asiático se suelen poner como ejemplo de minoría que, gracias a sus características culturales, prosperan social y económicamente mucho más que los negros ${ }^{5}$. Este tipo de teorías son comúnmente utilizadas para justificar el racismo y el sexismo más detestables, con el fin último de preservar y ampliar los privilegios del grupo beneficiario de la discriminación.

Sin embargo, otras formas de discriminación no se fundamentan en teorías elaboradas ni vienen precedidas de deliberaciones conscientes, sino que son el producto de procesos cognitivos inconscientes. En ese caso, se podría pensar que si no existe un propósito deliberado de discriminar, la discriminación no es mala por sí misma, sino en todo caso por sus efectos negativos, lo que parece que exculparía a los discriminadores de su actitud, aunque no impediría llevar a cabo acciones públicas contra la discriminación. Pero esa idea contraviene dos argumentos plausibles. En primer lugar, mantener un prejuicio por ignorancia no excluye la evaluación moral del prejuicio. Si éste es moralmente malo, lo sigue siendo tanto si su portador es consciente como si no. Y, en segundo lugar, la inconsciencia puede ser el producto de una inevitable irreflexión sobre las actitudes propias, como ocurre en los niños, pero también de una menos excusable falta de reflexión o de un sospechoso autoengaño, como ocurre con los adultos que hace mucho tiempo que ejercen la discriminación aun conociendo las teorías que la denuncian. En este último caso, la exculpación moral del discriminador es cuando menos discutible.

\footnotetext{
4 Sobre la importancia cognitiva y la racionalidad de las preferencias adaptativas, consúltese ELSTER, J., 1988: Uvas amargas, Barcelona: Península.

5 Véase, por ejemplo, Sowell, T., 1994: Race and Culture: a World View, New York: Basic Books; y Thernstrom, S., y Thernstrom, A., 1997: America in Black and White, New York: Simon and Schuster.
} 


\section{DISCRIMINACIÓN ENCUBIERTA}

Una de esas formas de discriminación inconsciente es la que podríamos denominar discriminación encubierta. Según ésta, algunas personas tratan a otras de manera diferente a partir de estereotipos o de esquemas cognitivos que estructuran sus percepciones y hábitos de conducta, a pesar de que ellas mismas desaprobarían tales representaciones si fuesen conscientes de su existencia. La psicología social y la sociología explican cómo se forman los estereotipos que conducen a los prejuicios y, finalmente, a la discriminación injusta. Los estereotipos son hábitos inconscientes de pensamiento que vinculan atributos personales a la pertenencia a un grupo. Pueden ser descriptivos o normativos. En el primer caso, son generalizaciones a partir de las percepciones que se tienen sobre cómo son los otros. Conducen a la discriminación cuando juzgamos a todos los miembros de un grupo a partir de los rasgos señalados por el estereotipo. En el segundo caso, son generalizaciones a partir de cómo suponemos que son los otros. Pueden basarse en el caso anterior, pero añaden ahora un juicio subjetivo que a menudo agudiza más la discriminación. Los estereotipos se acaban convirtiendo en teorías implícitas, llenas de prejuicios en la manera como percibimos, interpretamos, descodificamos y retenemos la información que recibimos de los otros. Están más relacionados con los procesos cognitivos que con las motivaciones. Por esa razón, lo curioso de los estereotipos es que están presentes incluso entre quienes los rechazarían de ser conscientes de su posesión, lo que hace que se pueda discriminar a los otros sin desear hacerlo.

Los estereotipos existen por una razón de eficiencia cognitiva. Según la psicología cognitiva ${ }^{6}$, ayudan a economizar la enorme y compleja información que recibimos del ambiente. Tienen como finalidad última la protección del estatus y los privilegios del grupo de referencia al que pertenecemos contra su posible pérdida por la injerencia de los otros, que también percibimos integrados en grupos. La categorización de las personas en grupos propios y ajenos es un proceso automático e inconsciente, y en este sentido el sexo, la etnia, el origen geográfico y nacional, las minusvalías o la clase social funcionan como grupos sociales claramente diferenciados y, por tanto, candidatos especiales a ser estereotipados. Por tal motivo, los estereotipos tienen un efecto de autoalimentación, puesto que los individuos acaban inconscientemente persiguiendo, prefiriendo y recordando información que apoya sus estereotipos, a la vez que ignoran, descartan y olvidan la información que los contradice. Cuanta más información tenemos que procesar y más urgente es su asimilación cognitiva, menos reflexión le dedicamos y más fácil acaba siendo la formación de estereotipos.

Pero los estereotipos son algo más que mecanismos de eficiencia cognitiva: producen prejuicios, es decir, evaluaciones sesgadas sobre las capacidades y el valor de los individuos que pertenecen a los grupos estereotipados. Un ejemplo lo ilustrará mejor que más teoría. Imaginémonos el estereotipo que dice que los hombres son mejores que las mujeres en aquellas tareas a las que tradicionalmente han estado más acostumbrados, como entender la mecánica de un coche. Según el estereotipo, a los

\footnotetext{
${ }^{6}$ Un interesante estudio sobre discriminación y psicología social se encuentra en FiSKE, S., 1998: «Stereotiping, Prejudice and Discrimination», en GILBERT, D.; FISKE, S., y LINDZEY, G. (eds.), Handbook of Social Psychology, New York: McGraw-Hill.
} 
hombres se les supone la competencia mecánica. De ese modo, si un hombre no consigue arreglar un motor renqueante o ni siquiera entender el mecanismo que ha producido una avería se suele achacar ese fracaso a factores relacionados con la situación, como la mala suerte o una falta de esfuerzo, sin que ello suponga una predicción futura sobre más fracasos en ese campo. En cambio, el estereotipo dice que las mujeres no están tan capacitadas para entender los misterios de mecánica de automoción, de manera que si también fracasan en el intento de arreglar o comprender el origen de la avería la explicación es mucho más fácil: carecen de la capacidad o de la habilidad necesaria para este tipo de conocimiento práctico y, por tanto, son previsibles más fracasos en el futuro. Y si resulta que tienen algún éxito, se explica por factores situacionales, como la buena suerte, que naturalmente no predice más éxitos en el futuro. Estereotipos como éste ayudan a explicar por qué las mujeres a menudo tienen que demostrar constantemente su capacidad profesional a pesar incluso de haber cosechado numerosos éxitos en su trabajo. Ellas deben demostrar no sólo que hacen algo bien, sino que también lo pueden hacer en el futuro, es decir, deben luchar contra los estereotipos habituales asociados a la capacidad desigual de hombres y mujeres para realizar trabajos tradicionales de hombres. Lo mismo ocurre con los estereotipos que padecen el resto de grupos discriminados en los diferentes ámbitos sociales y profesionales.

Los estereotipos están mucho más presentes en nuestras vidas y en nuestro pensamiento de lo que creemos, entre otras cosas porque son, como tales, el resultado inconsciente de los procesos cognitivos. Y muchos de los estereotipos esconden prejuicios innecesarios que conducen a discriminaciones injustas. Los prejuicios producidos por los estereotipos se afianzan en la estructura inconsciente del pensamiento con una fuerza que crea grandes resistencias a interpretar correctamente los hechos observados a través de ellos. No obstante, la ventaja de explicar parte de la discriminación a partir de los estereotipos es que, una vez que somos conscientes de ellos, tenemos la oportunidad de superarlos, lo que puede acabar reduciendo considerablemente el volumen actual de la discriminación. No sucede lo mismo con la discriminación consciente de quienes tienen el objetivo deliberado de preservar y ampliar sus privilegios. En ese caso, las estrategias de cambio deberían ser social y legalmente más contundentes.

Una característica especialmente relevante de los estereotipos desde el punto de vista de la discriminación es su correlación con el poder. Algunos estudios ${ }^{7}$ demuestran que las personas que disfrutan de mayor poder en la sociedad tienden a estereotipar más que aquellas acostumbradas a cumplir papeles sociales de subordinación. Estas últimas no se pueden permitir el lujo de estereotipar a aquellos de quienes dependen porque necesitan valorarlos con más cuidado, mientras que las personas que detentan mayor poder social estereotipan con mayor despreocupación a aquellos a quienes dominan, ya que su bienestar no depende de un conocimiento personalizado de sus subordinados. Eso explica por qué la discriminación es más difícil de vencer en las mentes de los privilegiados que en la de los subordinados, y es una razón más a favor

Operario, D.; Goodwin, S., y Fiske, S., 1998: «Power Is Everywhere: Social Control and Personal Control Both Operate at Stereotype Activation, Interpretation, and Inhibition», en WYER, R. (ed.), Stereotype Activation and Inbibition, Mahway, NJ: Lawrence Erlbaum Ass. 
de fundamentar el poder en convicciones igualitaristas que logren conectar los intereses de todos a través de un diálogo en igualdad de condiciones. El pensamiento igualitario tiene aquí la función instrumental de contribuir a deshacer los estereotipos que incomunican y desigualan injusta e innecesariamente a las personas.

\section{LA PARADOJA LIBERAL DE LA DISCRIMINACIÓN}

Por lo dicho hasta ahora, parecería que la discriminación depende completamente de la presencia de prejuicios sobre la inferioridad moral de determinados individuos o colectivos. Sin embargo, también aquí existen fronteras imprecisas. Algunas formas de discriminación hunden sus raíces en ideales morales particulares, en concepciones del bien, de la virtud y de lo que es una sociedad buena, sin que necesariamente contengan una teoría sobre la inferioridad de determinados grupos y personas, lo que dificulta tanto su evaluación moral como su erradicación. Si tales actitudes se juzgan como prejuicios, se podría pensar que esos juicios provienen de una concepción moral externa que el discriminador puede ignorar, o incluso despreciar, por considerarla ajena a su sistema de valores morales y culturales. Por ejemplo, algunas éticas religiosas reservan roles sociales diferentes para hombres y mujeres. Creen que las mujeres no deben dedicarse a la política, no pueden negarse a formar una familia o les está vedado participar en la vida pública con los mismos derechos que los demás. No es que piensen -necesariamente- que las mujeres están incapacitadas para ejercer los roles destinados a los hombres, pero creen que es inmoral que se salgan del papel social asignado por su religión. Otro ejemplo es el rol familiar reservado a los homosexuales en nuestra sociedad. Muchas personas opinan que las parejas homosexuales no deberían formar familias con hijos. Algunas de ellas pretenden basar sus creencias en teorías sobre el daño que sufrirían los hijos en un ambiente familiar homosexual, con lo cual la carga de la prueba recae en la racionalidad de la teoría. Pero otras simplemente consideran moralmente reprobable ese tipo de familia, aunque se demostrase que los hijos no padecen las calamidades psicológicas y sociales que algunos predicen.

Se puede objetar que tras esos juicios de valor sobre la conducta y los roles sexuales de las personas se esconde un prejuicio absoluto sobre la inferioridad moral de determinados individuos o colectivos. Si se niega a las mujeres el acceso al sacerdocio o a los homosexuales una paternidad responsable es porque existe un prejuicio encubierto sobre la inferioridad moral de las mujeres y los homosexuales respectivamente. Pero esa sospecha no es tan fácil de corroborar. Al fin y al cabo, mantener esos supuestos prejuicios es compatible con pensar que mujeres y homosexuales son perfectamente capaces de realizar cualquier otra tarea social. Esos juicios morales particulares o restringidos a los roles de género no necesariamente suponen la negación de la igualdad moral de las personas.

En cualquier caso, tanto si pensamos que esas creencias ocultan prejuicios absolutos como si no, lo cierto es que ese tipo de actitudes producen una inesperada paradoja en una sociedad liberal: cuanto más abierta y tolerante se muestra una sociedad con las diversas concepciones de la moralidad, más imprecisa se vuelve su capacidad para detectar, denunciar y prohibir las conductas basadas en ese tipo de creencias o juicios de valor, es decir, cuanto más liberal es la sociedad más difícil le resulta saber 
cuándo se está produciendo una discriminación basada en prejuicios. Si las discriminaciones se basan exclusivamente en prejuicios, las sociedades liberales, abiertas y tolerantes caen fácilmente en esa paradoja, puesto que cabe pensar que las creencias sobre la moralidad de los roles de género pueden no estar fundamentadas directamente en prejuicios, en "la negación de la igualdad entre los hombres", sino en ideales morales que, en principio, una sociedad tolerante con las diversas concepciones del bien debería permitir.

Una sociedad liberal es aquella donde todas las ideas del bien deben convivir en condiciones jurídicas de igualdad, de modo que se reconozca el derecho de todos a ejercer libremente sus ideales morales siempre que sean compatibles con los ideales de los demás. En caso de conflicto, la libertad de conciencia, de pensamiento y de vivir en el ámbito privado conforme a las propias convicciones debe prevalecer. Eso significa que, en una sociedad liberal, se debe permitir que los homosexuales adopten niños - siempre que no haya teorías contrastadas que demuestren un daño real a los menores - o que las mujeres accedan al poder político, al mismo tiempo que se tolera que existan grupos sociales que condenan moralmente la igualdad de género en esos ámbitos. Pero cuando la discriminación depende de concepciones morales del bien, y no de teorías contrastables sobre la supuesta inferioridad o incapacidad de las personas en función de su sexo o de estereotipos inconscientes que serían rechazados de asomar a la conciencia, resulta difícil confirmar la existencia de prejuicios. Naturalmente, se puede sospechar razonablemente que esos ideales morales esconden prejuicios, pero en una sociedad liberal y comprometida con el pluralismo moral no se dispone de más razones contra ellos de las que utilizaríamos para oponernos a la existencia de Dios. También se puede pensar que las personas que padecen la actitud de quienes defienden ideales morales discriminadores experimentan un oprobio y un insulto injustificables. Pero ése es el precio de pertenecer a una sociedad que respeta diferentes visiones del bien y del más allá. La ventaja de vivir en una sociedad tolerante, decía John Stuart MiLL, consiste, entre otras cosas, en que se minimiza el riesgo de haber escogido la creencia equivocada. Una sociedad tolerante no es aquella en que todas las actitudes morales correctas se acaban imponiendo socialmente, sino aquella otra en que todas esas actitudes reciben igual protección legal y política, es decir, no elimina la posibilidad de que el modo de vida personal escogido tenga detractores, pero tampoco permite que ninguna de esas detracciones impida a nadie seguir adelante con él.

Los prejuicios absolutos producen siempre discriminación, pero si consideramos que la discriminación es mala sólo cuando concurren prejuicios, podemos ser víctimas de la paradoja liberal de la discriminación antes apuntada. El antídoto contra esa paradoja consiste en rechazar al prejuicio como el designador rígido ${ }^{8}$ de la discriminación. Para apoyar ese rechazo, resulta pertinente traer a colación un tipo especial de discriminación que parece eludir la presencia de prejuicios: la discriminación estadística.

\footnotetext{
${ }^{8}$ En la teoría epistemológica del realismo esencialista, formulada por KRIPKE, S., el designador rígido (d.r.) describe la propiedad esencial de los objetos, lo que hace que podamos reconocer un objeto en cualquier contexto e independientemente de las propiedades accidentales que presente. Por ejemplo, $\mathrm{H}_{2} \mathrm{O}$ es el d.r. del agua: allí donde no hay $\mathrm{H}_{2} \mathrm{O}$ no hay agua. KRIPKE, S., 1992: El nombrar y la necesidad, México: UNAM.
} 


\section{DISCRIMINACIÓN ESTADÍSTICA}

La discriminación estadística se presenta cargada de racionalidad y no contiene necesariamente algún tipo de animadversión especial hacia los demás, lo que contrasta con la tesis de que es la presencia de prejuicios lo que vuelve moralmente inaceptable la discriminación ${ }^{9}$. Esta nueva forma de trato desigual y discriminatorio tiene que ver, por ejemplo, con el rendimiento productivo de los trabajadores en función del sexo y el origen étnico. Un empresario puede desarrollar dos tipos de creencias que conectan el sexo y el origen étnico con la adecuación para determinados empleos. En primer lugar, puede pensar que todas las mujeres o todas las personas de origen gitano, magrebí o asiático son inadecuadas - o al menos más inadecuadas que el resto- para ocupar determinados puestos de trabajo. En ese caso, lo más probable es que dicho empresario mantenga algún tipo de convicción sexista o racista sobre el valor de esas personas. Pero, en segundo lugar, puede creer que las mujeres y las personas de ciertas etnias o culturas tienden a ser menos adecuadas o productivas en determinados trabajos. Esta segunda creencia puede tener un fondo de prejuicio, como, por ejemplo, cuando se trata a todos los miembros de la etnia gitana de vagos e indisciplinados, o cuando se tacha a las mujeres de demasiado emocionales para ocupar puestos de responsabilidad profesional.

Pero en algunas ocasiones no está tan claro dónde está el prejuicio moral. Las compañías de seguros de automóvil suelen tener primas diferentes para hombres y mujeres, y para jóvenes y mayores. En este caso, la discriminación se defiende con el argumento de que los hombres jóvenes tienen más accidentes de tráfico o, lo que es lo mismo, conducen por regla general con más riesgo. Es obvio que detrás de este tipo de discriminación no hay un prejuicio moral, aunque se trata de manera diferente a las personas en función de su sexo y su edad, y sin importar si algunas mujeres mayores son, de hecho, más peligrosas al volante que algunos hombres jóvenes, siempre que su número sea reducido. Todos los miembros de un grupo son tratados por igual a partir de un comportamiento medio, sin una valoración individualizada. Pero la causa de la discriminación no reside necesariamente en un prejuicio, ni en la ignorancia, ni en la voluntad de defender determinados intereses de poder por parte de un colectivo privilegiado, sino en el deseo mucho más racional y aparentemente neutral de ser eficiente.

Imaginemos ahora un ejemplo diferente. Algunas personas opinan que los homosexuales no deberían formar parte del ejército, no porque la homosexualidad sea un

\footnotetext{
${ }^{9}$ La discriminación estadística puede ser confundida con la discriminación indirecta o disparate impact, pero no son lo mismo. Esta última no exige la intencionalidad del discriminador como elemento probatorio de la discriminación (a diferencia de la discriminación directa o disparate treatment) y, por tanto, utiliza los efectos de la conducta para evaluar la discriminación y no sus razones. Pero presupone la existencia de razones prejuiciosas. Si no las tiene en cuenta para comprobar la discriminación se debe a que resulta técnica o legalmente muy difícil hacerlo. Es difícil comprobar, por ejemplo, que un empresario mantiene intencionalmente un prejuicio sexista si no promociona a una determinada mujer dentro de su empresa por el hecho de ser mujer. Puede camuflar su prejuicio con el establecimiento de requisitos y condiciones de acceso de apariencia neutral. Es más fácil presuponer ese prejuicio si se comprueba que general o sistemáticamente promociona a los hombres y no a las mujeres. La peculiaridad de la discriminación estadística es que, en ocasiones, ni siquiera cabe presuponer un prejuicio moral absoluto (basado en la inferioridad moral de determinados individuos o colectivos) para reconocer la discriminación.
} 
impedimento para convertirse en un excelente soldado, sino porque la convivencia en los cuarteles entre homosexuales y heterosexuales genera unas incomodidades que reducen, en general, la armonía y el compañerismo imprescindibles para el buen hacer de un ejército. ¿Se trata, en este caso, de un prejuicio que debe ser eliminado o de una razón pertinente que hay que tomar en consideración para prohibir el ingreso de homosexuales en el ejército? Imaginemos ahora a un empresario que necesita operarios para trabajar en una fábrica textil. De entre todos los candidatos presentados, mayoritariamente mujeres, rechaza sistemáticamente a los de origen magrebí. La razón es que, en otras ocasiones, ha contratado a mujeres norteafricanas, pero se ha dado cuenta de que tienen un índice de absentismo laboral muy elevado en comparación con las demás. Naturalmente, siempre había alguna excepción, incluido el absentismo laboral reiterado de algunas mujeres de otras etnias. Como que, antes de realizar los contratos, no puede prever qué individuos realmente tendrán un absentismo laboral elevado y cuáles no, simplemente excluye a las norteafricanas de futuras contrataciones. Según razona el empresario, es una decisión dictada por la eficiencia, ya que lo contrario sería tomar decisiones contra su negocio. Se niega a pensar que es un acto de racismo. De hecho, su hijo está saliendo con una joven marroquí y en casa están encantados con la muchacha. ¿Existe en este caso una discriminación laboral injusta?

Podríamos pensar que es injusto tratar a todos los individuos según el comportamiento medio de su grupo, porque eso los despersonaliza, es decir, impide que su valía profesional dependa de ellos mismos, de su esfuerzo y capacidad individuales, de su mérito personal. Las personas merecen un respeto individual, independientemente de si son miembros de un colectivo u otro. «Cualquier individuo perteneciente a un grupo determinado que es víctima de una discriminación estadística nunca habría tenido una oportunidad para competir, para probar su capacidad. Sería juzgado completamente sobre la base de la conducta de otras personas — personas que comparten algunas características arbitrarias con él一 ${ }^{10}$. Este argumento es realmente plausible, propio de las éticas de principios o deontológicas —aunque la ética utilitarista más seria tampoco renuncia al individualismo ético, a pesar de que en los casos de justicia distributiva priorice sistemáticamente el bienestar colectivo al individual- ${ }^{11}$. Entonces, ¿hemos dado ya con la razón que condena cualquier tipo de discriminación estadística?

Vamos a comprobar que las dudas permanecen. La selección de las personas a través del mérito responde a la misma lógica de la discriminación estadística. En muchas ocasiones, los individuos no pueden demostrar que son aptos para realizar un trabajo antes de realizarlo - e incluso antes de acumular cierta experiencia sobre él一. Lo que el principio del mérito hace es seleccionar, a través de alguna prueba objetiva, a los individuos que previsiblemente desarrollarán mejor la tarea para la que son seleccionados, ya que la estadística muestra que los que alcanzan determinada puntuación en las pruebas objetivas tienen más probabilidades de hacer mejor el trabajo. Es imposi-

${ }^{10}$ Fishrin, J., 1983: Justice, Equal Opportunity, and the Family, New Haven: Yale University Press, p. 24. Una tesis contraria a la de FishKIN se puede encontrar en CAVANAGH, M., 2002: Against Equality of Opportunity, Oxford: Clarendom Press, Part 3.

${ }_{11}$ Para una defensa del individualismo ético en el utilitarismo, véase, por ejemplo, SINGER, P., 1991: Ética práctica, Barcelona: Ariel. 
ble individualizar totalmente el mérito. Puede ocurrir que no se disponga del tiempo y los mecanismos necesarios para ello, que los costes sean desproporcionados, y que sencillamente resulte imposible, ya que a menudo parte del mérito sólo se puede demostrar una vez se ha empezado a trabajar, o incluso tiempo después. Si fuese posible una completa personalización del mérito, muchos empresarios descubrirían que algunos de los aspirantes descartados por la prueba objetiva tenían unas cualidades ocultas mejores que las de los elegidos inicialmente. En cualquier caso, la selección a través del mérito es ya un claro ejemplo de discriminación estadística que, por supuesto, no censuramos moralmente.

Se podría replicar que el mérito incluye factores que las personas pueden controlar, como el esfuerzo y la ambición, mientras que si el factor determinante para ser rechazado en el acceso a un trabajo es el sexo o el origen étnico y cultural, se está juzgando a los individuos por factores que éstos no pueden controlar y de los cuales, por tanto, no se les puede responsabilizar. Y es precisamente eso - la violación del principio de responsabilidad, y no del principio del mérito- lo que vuelve injusta a la discriminación estadística. Sin embargo, si seguimos con esa argumentación, también habría que impedir a los empresarios que contratasen a sus trabajadores basándose en un factor también moralmente arbitrario como la inteligencia natural. La inteligencia natural, como el sexo o el origen étnico, es un atributo que cae fuera de la responsabilidad de los individuos, y si lo que convierte a la discriminación estadística en inmoral es que utiliza ese tipo de atributos para juzgar la adecuación de los individuos al trabajo, entonces también habría que señalar como inmoral la contratación de un profesional a partir de su inteligencia natural. Si no lo hacemos, si no creemos que el caso de la inteligencia sea moralmente censurable en la selección de los candidatos a un puesto de trabajo, es porque la inteligencia señala un factor de eficiencia. Las personas más inteligentes presumiblemente realizarán mejor los trabajos complejos que los menos inteligentes, como las personas con una determinada habilidad natural tienen más probabilidades de realizar mejor las tareas que exigen ese tipo de habilidad, y ésa es la razón por la que tienen prioridad en el acceso a algunos trabajos.

Aún se podría replicar que la inteligencia o las habilidades naturales no son nada por sí mismas y que siempre requieren de una cierta responsabilidad del individuo para desarrollarlas, cosa que es imposible si partimos del sexo o el origen étnico. Pero entonces también deberíamos admitir que los empresarios que condicionan el acceso a un trabajo al hecho de poseer un determinado acento y unas maneras de vestir no están discriminando en función de la clase social, a pesar de que en la práctica sólo los miembros de una determinada clase social cumplan los requisitos impuestos por el empresario. Todos tenemos la posibilidad, ni que sea remota en muchos casos, de reeducar el acento y de adecuar la forma de vestir a cualquier exigencia social. Sin embargo, no creo que esa mera posibilidad sea suficiente para volver aceptable la política de selección del empresario clasista. Por tanto, el principio de la responsabilidad individual no basta para censurar moralmente la discriminación estadística.

Ahora bien, que la discriminación estadística, incluso si utiliza el sexo o el origen étnico y cultural como criterio de exclusión, no sea siempre moralmente condenable, ya que no contiene necesariamente actitudes prejuiciosas inaceptables, no significa que debamos despreocuparnos de sus efectos morales negativos. Por un lado, algunos empresarios pueden utilizar la discriminación estadística para encubrir prejuicios into- 
lerables. Cuando se rechaza a mujeres o a africanos como candidatos a desarrollar un trabajo de cara al público alegando que una parte significativa de la clientela se muestra reacia a tratar con personas de su condición, el empresario se convierte en vehículo del sexismo y el racismo, y entonces debemos condenarlo y poner los medios para impedirlo. Cuando se rehúsa contratar a mujeres jóvenes por miedo a que los embarazos posibles perjudiquen la productividad de la empresa, también es necesario intervenir, por ejemplo a través de ayudas públicas tanto a mujeres embarazadas como a los empresarios que las contratan para que esa circunstancia provoque los menores costes de productividad posibles. Por otro lado, puede ocurrir que, simplemente, algunos individuos y colectivos vean disminuidas sus oportunidades laborales y sociales debido a la discriminación estadística, lo que todavía es más grave si los discriminados son los colectivos históricamente perjudicados por la discriminación inmoral. También aquí los poderes públicos tienen la obligación de intervenir. Una forma de hacerlo es a través de una presión fiscal especial a las empresas que utilizan la discriminación estadística para obtener mayores beneficios económicos. Se trataría de que los beneficios económicos de la discriminación estadística acabasen favoreciendo a todos, incluidos los discriminados. Otra medida es limitar legalmente las ocasiones en que se permite la discriminación estadística, con el fin de reducirla a los casos que presentan menos sospechas de discriminación inmoral encubierta.

En conclusión, a pesar de que la discriminación estadística no siempre está vinculada a un prejuicio moral contra las personas discriminadas, es igualmente condenable desde la ética. Porque lo que convierte en mala a la discriminación no lo debemos buscar en el punto de vista de quien discrimina, sino en el efecto de su acción sobre la persona discriminada. La perspectiva de la víctima, su falta o su recorte de oportunidades por circunstancias que le son ajenas, es lo que la ética de la igualdad de oportunidades debe denunciar. Otra cosa es la condena moral - e incluso penal- que merece el discriminador, que, sin duda, debe ser proporcional a su responsabilidad, pero no es necesario juzgar sus ideas o intenciones —si contienen o no prejuicios, si se fundamenta o no en ideas inmorales...- para identificar una discriminación como mala. Los motivos del discriminador son importantes para juzgar su conducta y para descubrir los motivos ocultos de ésta, pero son las consecuencias de su acción, el perjuicio que ésta causa en la víctima —incluida la humillación-, lo que vuelve intolerable como tal la discriminación. Las teorías racistas y sexistas o el simple menosprecio contra las personas por su sexo, etnia o discapacidad son moralmente reprobables, pero es el recorte de oportunidades iguales que se derivan de las actitudes discriminatorias lo que convierte en mala la discriminación y lo que resulta condenable desde una ética igualitarista.

\section{$\triangle \quad$ DOXA 29 (2006)}

